\title{
On the Convergence of Collocation Methods for Boundary Integral Equations on Polygons
}

\author{
By Martin Costabel and Ernst P. Stephan*
}

\begin{abstract}
The integral equations encountered in boundary element methods are frequently solved numerically using collocation with spline trial functions. Convergence proofs and error estimates for these approximation methods have been only available in the following cases: Fredholm integral equations of the second kind [4], [7], one-dimensional pseudodifferential equations and singular integral equations with piecewise smooth coefficients on smooth curves [2], [3], [17], [26]-[29], and some special results on the classical Neumann integral equation of potential theory for polygonal plane domains [5], [8], [9]. Here we give convergence proofs for collocation with piecewise linear trial functions for Neumann's integral equation and Symm's integral equation on plane curves with corners. We derive asymptotic error estimates in Sobolev norms and analyze the effect of graded meshes.
\end{abstract}

0. Introduction. In this paper we give convergence proofs and asymptotic error estimates in Sobolev norms for collocation with piecewise linear spline trial functions applied to two basic integral equations of potential theory on plane polygons, namely the integral equation of the second kind with the double layer potential ("Neumann's integral equation"), and the integral equation of the first kind with the simple layer potential ("Symm's integral equation"). We use an idea of Arnold and Wendland [2], namely considering Dirac delta functions (the "test functions" in the collocation method) as second derivatives of piecewise linear functions. Therefore, similar results as presented here should be possible for splines of higher odd order. Corresponding results for even-order splines are not yet available. Thus, for one of the simplest methods of numerically solving Dirichlet's problem on a plane domain with corners, the midpoint collocation with piecewise constant trial functions for the first-kind integral equation with the simple layer potential, convergence is still an open problem. The method of Fourier series that yields the convergence proof in the case of a smooth boundary [27] cannot be applied in the presence of corners.

We apply the method of local Mellin transformation that has previously been used to derive error estimates for Galerkin methods for a wide class of operators, including those occurring in boundary element methods in acoustics, electromagnetism, and elastostatics [11]-[14]. Thus, it is to be expected that also the techniques presented here will apply to a rather large class of integral equations. For example,

Received June 9, 1986.

1980 Mathematics Subject Classification (1985 Revision). Primary 65N35, 65R20; Secondary 65D07, $45 \mathrm{~L} 10$.

* This author was supported by the National Science Foundation under Grant DMS-8501797 and Grant DMS-8603954. 
the case of singular integral equations of Cauchy type will be treated in a forthcoming paper.

Let $\Gamma$ be a connected closed plane curve composed of smooth $\operatorname{arcs} \Gamma^{j}, j=1, \ldots, J$, that meet' at the corner points $z_{j}$ at interior angles $\omega_{j} \in(0,2 \pi)$. The Sobolev spaces $H^{s}(\Gamma)$ are defined for $s>0$ being the restriction of $H^{s+1 / 2}\left(\mathbf{R}^{2}\right)$ to $\Gamma$, for $s<0$ by duality: $H^{s}(\Gamma)=H^{-s}(\Gamma)^{\prime}$, and $H^{0}(\Gamma)=L^{2}(\Gamma)$. It is known [11], [12] that for $|s|<3 / 2$, the space $H^{s}(\Gamma)$ may equivalently be defined as the corresponding Sobolev space on the arc length parameter interval, transferred to $\Gamma$ by the parameter representation map.

We consider the following two integral equations on $\Gamma$ :

$$
\begin{gathered}
(1+K) u=f, \\
V u=f .
\end{gathered}
$$

Here the operator $K$ of the double layer potential is defined by

$$
K u(z):=-\frac{1}{\pi} \int_{\Gamma} u(\zeta) \frac{\partial}{\partial n(\zeta)} \log |z-\zeta| d s(\zeta),
$$

where $s(\zeta)$ is the arc length on $\Gamma$ and $\partial / \partial n(\zeta)$ denotes the derivative with respect to the normal vector at $\zeta \in \Gamma$ pointing into the interior of $\Gamma$. The operator $V$ of the simple layer potential is defined by

$$
V u(\zeta)=-\frac{1}{\pi} \int_{\Gamma} u(\zeta) \log |z-\zeta| d s(\zeta)
$$

It is known [11] that $1+K: H^{s}(\Gamma) \rightarrow H^{s}(\Gamma)$ is continuous and bijective for all $s \in\left(\frac{1}{2}-\alpha_{0}, \frac{1}{2}+\alpha_{0}\right)$, where we define $\alpha_{0}, \alpha_{1}, \ldots, \alpha_{J} \in\left(\frac{1}{2}, 1\right)$ by

$$
\alpha_{j}:=\min \left\{\frac{\pi}{\omega_{j}}, \frac{\pi}{2 \pi-\omega_{j}}\right\} ; \quad \alpha_{0}:=\min \left\{\alpha_{j} \mid j=1, \ldots, J\right\} .
$$

Similarly, $V: H^{s}(\Gamma) \rightarrow H^{s+1}(\Gamma)$ is continuous and bijective for all $s \in\left(-\frac{1}{2}-\alpha_{0}\right.$, $\left.-\frac{1}{2}+\alpha_{0}\right)$, provided the analytic capacity of $\Gamma$ is not equal to one. We shall assume this in the sequel.

For the collocation method, we need a grid $\Delta_{N}=\left\{x_{1}, \ldots, x_{N}\right\} \subset \Gamma$, the $x_{j}$ being both the collocation points and the meshpoints of the trial functions. By $S^{1}\left(\Delta_{N}\right)$ we denote the $N$-dimensional space of splines of order 1, i.e., each $u \in S^{1}\left(\Delta_{N}\right)$ is a continuous function on $\Gamma$ that is a linear function of the arc length on each of the segments ${\widetilde{x_{n} x}}_{n+1}, n=0, \ldots, N-1$, where $x_{0}:=x_{N}$. Let

$$
h:=\max \left\{\left|x_{n+1}-x_{n}\right| \mid n=0, \ldots, N-1\right\} .
$$

We do not impose a uniformity condition on $\Delta_{N}$, but assume only that $h \rightarrow 0$ as $N$ tends to infinity.

For the second-kind integral equation (0.1), the collocation method is the following:

Find $u_{N} \in S^{1}\left(\Delta_{N}\right)$ such that

$$
(1+K) u_{N}\left(x_{n}\right)=f\left(x_{n}\right), \quad n=1, \ldots, N .
$$

For the first-kind integral equation (0.2), the collocation equations are

$$
V u_{N}\left(x_{n}\right)=f\left(x_{n}\right), \quad n=1, \ldots, N,
$$


but we shall have to modify (0.6) in some way in order to obtain a convergence proof (see (3.3) and (3.6)).

The paper is organized as follows: In Section 1 we present some facts on the convergence of general projection methods. They are stated in a form which is convenient for the application to collocation methods and allow easy incorporation of compact perturbations as well as localization arguments.

In Section 2 we prove convergence and stability in the $H^{1}$ Sobolev norms for the approximation scheme $(0.5)$ for the second-kind integral equation $(0.1)$.

In Section 3 convergence and stability results for two modifications of the scheme (0.6) for the first-kind integral equation (0.2) are shown.

In the final Section 4 we investigate the asymptotic orders of convergence. For the case of the first-kind integral equation, where we have to use weighted Sobolev norms, we prove a new approximation result and we show that the use of suitably graded meshes yields convergence of the same order as for smooth curves.

1. On the Convergence of Projection Methods. We need some results on the convergence of projection methods, including compact perturbations and spaces with two norms. Such results are well known [16], [19], [24], but we present a formulation that is particularly adapted to the present case. As the lemma in question might be of independent interest, we also include a complete proof.

Let $X$ and $Y$ be Banach spaces and $A: X \rightarrow Y$ be bijective and continuous. For the approximate solution of the equation

$$
A u=f
$$

we assume that we have a sequence of finite-dimensional subspaces

$$
V_{N} \subset X, \quad T_{N} \subset Y^{\prime}, \quad \operatorname{dim} V_{N}=\operatorname{dim} T_{N}<\infty \quad(N \in \mathbf{N})
$$

and we replace Eq. (1.1) by the relation for $u_{N} \in V_{N}$,

$$
\left\langle t, A u_{N}\right\rangle=\langle t, f\rangle \quad \text { for all } t \in T_{N} .
$$

Here the brackets denote the duality between the space $Y$ and its dual $Y^{\prime}$. We make the following assumptions:

(i) There exist bounded operators $P_{N}: Y^{\prime} \rightarrow T_{N}$ that converge on $Y^{\prime}$ strongly to the identity operator.

(ii) There is a Banach space $X_{0}$, continuously embedded in $X$ (hence, $\|x\|_{X} \leqslant$ $C\|x\|_{X_{0}}$ for all $x \in X_{0}$ and some constant $C$ ).

(iii) For all $N$ there holds $V_{N} \subset X_{0}$.

(iv) For all $N$ we are given a mapping $Q_{N}: V_{N} \rightarrow T_{N}$ and a constant $M$ such that

$$
\left|\left\langle Q_{N} v, A w\right\rangle\right| \leqslant M\|v\|_{X}\|w\|_{X_{0}} \text { for all } v \in V_{N}, w \in X_{0}, N \in \mathbf{N} \text {. }
$$

(v) There exists a collectively compact sequence of operators $C_{N}: X \rightarrow X^{\prime}$ in the sense of [1] and a constant $\gamma>0$ such that

$$
\left|\left\langle Q_{N} v, A v\right\rangle+\left\langle C_{N} v, v\right\rangle\right| \geqslant \gamma\|v\|_{X}^{2} \text { for all } v \in V_{N}, N \in \mathbf{N}
$$

LEMma 1.1. Under the above conditions (i)-(v) there exists $N_{0} \in \mathbf{N}$ such that for all $N \geqslant N_{0}$ the system (1.2) has a unique solution $u_{N} \in V_{N}$ for any $f \in Y$. There is a constant $C$ such that for this "approximate solution" $u_{N}$ and the "true solution" $u$ 
there holds

$$
\begin{gathered}
\left\|u_{N}\right\|_{X} \leqslant C\|u\|_{X_{0}} \quad \text { for all } u \in X_{0}, N \geqslant N_{0} \\
\left\|u-u_{N}\right\|_{X} \leqslant C \inf \left\{\|u-\tilde{u}\| x_{0} \mid \tilde{u} \in V_{N}\right\} .
\end{gathered}
$$

Proof. For abbreviation, we write $\|\cdot\|:=\|\cdot\|_{X}$ and $\|\cdot\|_{0}:=\|\cdot\|_{X_{0}}$. The derivation of the quasi optimality (1.6) from the stability (1.5) and the unique solvability of the system (1.2) is standard:

Denote the solution operator $u \mapsto u_{N}$ by $G_{N}$. Then $G_{N}: X_{0} \rightarrow\left(V_{N},\|\cdot\|\right)$ is a projection operator. Its norm is bounded by $C$ for all $N \geqslant N_{0}$ by (1.5). Thus for all $\tilde{u} \in V_{N}$ :

$$
\left\|u-u_{N}\right\|=\left\|u-\tilde{u}-G_{N}(u-\tilde{u})\right\| \leqslant\|u-\tilde{u}\|+C\|u-\tilde{u}\|_{0} .
$$

Hence the assertion (1.6) follows.

For the proof of unique solvability of (1.2) and stability estimate (1.5) we consider first the special case where all operators $C_{N}$ in assumption (v) vanish. (Actually, this is not so special: The existence of $Q_{N}$ with (1.4) for $C_{N} \equiv 0$ is also necessary for stability, cf. [17].) Then from (1.4) it follows that the solution of (1.2) is unique, namely:

$$
\begin{aligned}
& \text { If }\langle t, A v\rangle=0 \text { for all } t \in T_{N} \text { and some } v \in V_{N}, \text { then } \\
& \gamma\|v\|^{2} \leqslant\left|\left\langle Q_{N} v, A v\right\rangle\right|=0, \text { hence } v=0 .
\end{aligned}
$$

As (1.2) is represented, after choosing bases in $V_{N}$ and $T_{N}$, by an $N \times N$ system of equations, the existence of $u_{N}$ follows from uniqueness. The stability estimate (1.5) follows from (1.2), (1.3), (1.4):

$$
\left\|u_{N}\right\|^{2} \leqslant \frac{1}{\gamma}\left|\left\langle Q_{N} u_{N}, A u_{N}\right\rangle\right|=\frac{1}{\gamma}\left|\left\langle Q_{N} u_{N}, A u\right\rangle\right| \leqslant \frac{M}{\gamma}\left\|u_{N}\right\|\|u\|_{0} .
$$

Now we consider the general case with nonvanishing perturbations $C_{N}$. We define new operators $\tilde{Q}_{N}$ and show that all assumptions are satisfied for large $N$ if we replace $Q_{N}$ by $\tilde{Q}_{N}$ and $C_{N}$ by 0 . Thus we reduce the general case to the special case considered above. Define

$$
\tilde{Q}_{N}:=Q_{N}+P_{N} A^{\prime-1} C_{N}=Q_{N}+A^{\prime-1} C_{N}-\left(1-P_{N}\right) A^{\prime-1} C_{N} .
$$

Here $A^{\prime-1}$ is the inverse of the isomorphism $A^{\prime}: Y^{\prime} \rightarrow X^{\prime}$ adjoint to $A$. The norms of $P_{N} A^{\prime-1} C_{N}: X \rightarrow Y^{\prime}$ are bounded, hence

$$
\begin{aligned}
\left|\left\langle\tilde{Q}_{N} v, A w\right\rangle\right| & \leqslant\left|\left\langle Q_{N} v, A w\right\rangle\right|+\left|\left\langle P_{N} A^{\prime-1} C_{N} v, A w\right\rangle\right| \\
& \leqslant M\|v\|\|w\|_{0}+M_{1}\|v\|\|w\| \\
& \leqslant\left(M+C M_{1}\right)\|v\|\|w\|_{0} .
\end{aligned}
$$

Thus (1.3) holds for $\tilde{Q}_{N}$ and all $N$. As $1-P_{N} \rightarrow 0$ strongly on $Y^{\prime}$ and the operators $A^{\prime-1} C_{N}: X \rightarrow Y^{\prime}$ are collectively compact, $A^{\prime}\left(1-P_{N}\right) A^{\prime-1} C_{N}: X \rightarrow X^{\prime}$ tends to zero in operator norm. If we denote the operator norm of $A^{\prime}\left(1-P_{N}\right) A^{\prime-1} C_{N}$ by $\delta_{N}$, 
we obtain

$$
\begin{aligned}
\left|\left\langle\tilde{Q}_{N} v, A v\right\rangle\right| & =\left|\left\langle Q_{N} v, A v\right\rangle+\left\langle A^{\prime-1} C_{N} v, A v\right\rangle+\left\langle\left(1-P_{N}\right) A^{\prime-1} C_{N} v, A v\right\rangle\right| \\
& =\left|\left\langle Q_{N} v, A v\right\rangle+\left\langle C_{N} v, v\right\rangle+\left\langle A^{\prime}\left(1-P_{N}\right) A^{\prime-1} C_{N} v, v\right\rangle\right| \\
& \geqslant\left(\gamma-\delta_{N}\right)\|v\|^{2} .
\end{aligned}
$$

Thus, if $N$ is large enough to imply $\delta_{N}<\gamma$, the corresponding estimate (1.4) holds for $\tilde{Q}_{N}$, and $C_{N}$ replaced by zero. This completes the proof.

Remark 1.2. We shall need the lemma only for the case of $Q_{N}$ and $C_{N}$ not depending on $N$. Thus $Q_{N} \equiv Q: X_{0} \rightarrow Y^{\prime}$ will be a linear operator satisfying

$$
Q V_{N} \subset T_{N} \text { for all } N \in \mathbf{N} \text {, }
$$

and $C_{N} \equiv C: X \rightarrow X^{\prime}$ will be a compact operator, or equivalently, the quadratic form $v \mapsto\langle C v, v\rangle$ appearing in (1.4) will be completely continuous on $X$.

Remark 1.3. The operators $P_{N}: Y^{\prime} \rightarrow T_{N}$ are not explicitly needed for (1.2). Only their existence is used in the proof. If $Y^{\prime}$ is a Hilbert space, we can take the orthogonal projections onto $T_{N}$. We then must assume that $T_{N} \rightarrow Y^{\prime}$ in the sense that for all $t \in Y^{\prime}$ there is a sequence $t_{N} \in T_{N}$ converging to $t$. By duality and the reflexivity of $Y$, this can be formulated as the following condition.

$$
\begin{aligned}
& \text { If } y \in Y \text { and } \lim _{N \rightarrow \infty}\left\langle t_{N}, y\right\rangle=0 \text { for each sequence } t_{N} \in T_{N}, \\
& \text { then } y=0 \text {. }
\end{aligned}
$$

We shall use this condition later on instead of (i) above.

The Garding type inequality (1.4) can be localized by means of a partition of unity. We formulate this result for the situation of spaces with two norms but with $Q_{N}$ and $C_{N}$ not depending on $N$. Thus, we make the following assumptions: $Q$ : $X_{0} \rightarrow Y^{\prime}$ is a linear operator with $A^{\prime} Q: X \rightarrow X_{0}^{\prime}$ bounded (according to (1.3)). There exist bounded linear commuting operators $a_{j}(j=1, \ldots, m)$ on $X$ and $b_{j}$ $(j=1, \ldots, m)$ on $Y$ such that

( $\alpha$ ) $\sum_{j=1}^{m} a_{j}^{2}=1$ on $X$;

( $\beta$ ) $B_{j}^{0}:=A a_{j}-b_{j} A$ is compact from $X$ to $A X_{0} \subset Y$; i.e., $A^{-1} B_{j}^{0}: X \rightarrow X_{0}$ is compact;

( $\gamma$ ) $B_{j}^{1}:=Q a_{j}-b_{j}^{\prime} Q$ is compact from $X$ to $Y^{\prime}$

( $\delta$ ) For every $k=1, \ldots, m$ there is a compact operator $C_{k}: X \rightarrow X^{\prime}$ and a constant $\gamma_{k}>0$ such that

$$
\operatorname{Re}\left(\left\langle Q a_{k} v, A a_{k} v\right\rangle+\left\langle C_{k} v, v\right\rangle\right) \geqslant \gamma_{k}\left\|a_{k} v\right\|_{X}^{2} \quad \text { for all } v \in X .
$$

LEMMA 1.4. Let the assumptions $(\alpha)-(\delta)$ be satisfied. Then there exists a compact operator $C: X \rightarrow X^{\prime}$ and a constant $\gamma>0$ such that

$$
\operatorname{Re}(\langle Q v, A v\rangle+\langle C v, v\rangle) \geqslant \gamma\|v\|_{X}^{2} \quad \text { for all } v \in X
$$

In particular, (1.4) holds.

Proof. From $(\beta)$ and $(\gamma)$ it follows that for $j, k=1, \ldots, m$,

$$
\left\langle Q a_{j}^{2} v, A a_{k}^{2} v\right\rangle=\left\langle Q a_{k} a_{j} v, A a_{k} a_{j} v\right\rangle+\left\langle C_{k j} v, v\right\rangle
$$


with $C_{k j}: X \rightarrow X^{\prime}$ compact. Here one has to use that $B_{j}^{0 \prime} Q: X \rightarrow X^{\prime}$ is compact. There follows

$$
\begin{aligned}
\langle Q v, A v\rangle & =\sum_{j, k=1}^{m}\left\langle Q a_{j}^{2} v, A a_{k}^{2} v\right\rangle \\
& =\sum_{j, k=1}^{m}\left(\left\langle Q a_{k} a_{j} v, A a_{k} a_{j} v\right\rangle+\left\langle C_{k j} v, v\right\rangle\right) .
\end{aligned}
$$

By (1.9),

$$
\operatorname{Re}\left(\left\langle Q a_{k} a_{j} v, A a_{k} a_{j} v\right\rangle+\left\langle C_{k} a_{j} v, a_{j} v\right\rangle\right) \geqslant \gamma_{k}\left\|a_{k} a_{j} v\right\|^{2},
$$

hence

$$
\begin{aligned}
\operatorname{Re}\langle Q v, A v\rangle & \geqslant \sum_{j, k=1}^{m}\left(\gamma_{k}\left\|a_{k} a_{j} v\right\|^{2}-\operatorname{Re}\left\langle C_{k j} v, v\right\rangle-\operatorname{Re}\left\langle C_{k} a_{j} v, a_{j} v\right\rangle\right) \\
& \geqslant \gamma\|v\|^{2}-\operatorname{Re}\langle C v, v\rangle
\end{aligned}
$$

with $\gamma:=\frac{1}{m} \min \left\{\gamma_{k} \mid k=1, \ldots, m\right\}$ and $C=\sum_{j, k}\left(C_{k j}+a_{j}^{\prime} C_{k} a_{j}\right)$.

Remark 1.5. Note that in this formulation the finite-dimensional spaces $V_{N}$ and $T_{N}$ do not appear. Therefore, this local principle is very easy to apply. Compare also the local principles of Prössdorf [22] and Silbermann [17].

2. The Second-Kind Integral Equation. In order to show convergence of the collocation scheme (0.5) for the integral equation (0.1), we apply Lemma 1.1 to the following situation: $X_{0}=X=Y=H^{1}(\Gamma) ; A=1+K ; \quad V_{N}:=S^{1}\left(\Delta_{N}\right)$; $T_{N}:=S^{-1}\left(\Delta_{N}\right)$, where

$$
S^{-1}\left(\Delta_{N}\right):=\operatorname{span}\left\{\delta\left(x-x_{n}\right) \mid n=1, \ldots, N\right\} .
$$

We have to check the assumptions of Lemma 1.1.

The abstract Galerkin equations (1.2) coincide with the collocation equations (0.5). Furthermore, assumption (i) of Lemma 1.1 is satisfied in view of Remark 1.3. Namely, $H^{1}(\Gamma)$ is continuously embedded into $C(\Gamma)$ and the condition $h \rightarrow 0$ implies that every point $\zeta \in \Gamma$ is an accumulation point of a sequence $\zeta_{N} \in \Delta_{N}$. Thus the hypothesis in condition (1.8) is only satisfied if $y \equiv 0$ on $\Gamma$.

Next we define

$$
Q:=D^{2},
$$

i.e., the second distributional derivative with respect to the arc length. Then clearly

$$
Q S^{1}\left(\Delta_{N}\right) \subset S^{-1}\left(\Delta_{N}\right)
$$

holds, i.e., (1.7) is satisfied. Note that our lemma does not require $Q_{N}$ to be bijective! (The latter property is frequently assumed in other approaches [2].)

We have to show the two estimates (1.3), (1.4) in the case $Q_{N}=Q$. The first one follows by continuity: Let $v, w \in H^{1}(\Gamma)$; then

$$
\begin{aligned}
|\langle Q v,(1+K) w\rangle| & =\left|-\langle D v, D(1+K) w\rangle_{L^{2}(\Gamma)}\right| \\
& \leqslant\|v\|_{H^{1}(\Gamma)}\|(1+K) w\|_{H^{1}(\Gamma)} \leqslant M\|v\|_{H^{1}(\Gamma)}\|w\|_{H^{1}(\Gamma)} .
\end{aligned}
$$

Note (2.4) is true for any Lipschitz curve $\Gamma$. 
The Gårding type estimate (1.4) requires more work, and it is only here that we use the special shape of $\Gamma$ as a (curved) polygon.

LEMMA 2.1. There is a constant $\gamma>0$ and a compact operator $C: H^{1}(\Gamma) \rightarrow H^{-1}(\Gamma)$ such that

$$
|\langle D v, D(1+K) v\rangle+\langle C v, v\rangle| \geqslant \gamma\|v\|_{H^{1}(\Gamma)}^{2} \quad \text { for all } v \in H^{1}(\Gamma) .
$$

For the proof of (2.5) we use a partition of unity and Lemma 1.4 to reduce (2.5) to the corresponding estimate on a reference angle.

Let $\Gamma_{\omega}=e^{i \omega} \mathbf{R}_{+} \cup \overline{\mathbf{R}_{+}}$be this reference angle. If we use $\Gamma_{\omega}$ to parametrize a neighborhood of one of the corners $z_{j}$, then the operator induced by $K$ differs from the operator of the double layer potential defined on $\Gamma_{\omega}$ only by an operator that is compact on $H^{1}$; see [10]. Thus we only need to consider the case that $\Gamma$ and $\Gamma_{\omega}$ coincide on a neighborhood of the origin, and $K$ is defined on $\Gamma_{\omega}$. We then have to show

LEMMA 2.2. There is $\gamma>0$ such that

$$
\operatorname{Re}\langle D v, D(1+K) v\rangle \geqslant \gamma\|v\|_{H^{1}\left(\Gamma_{\omega}\right)}^{2}
$$

for all $v \in H^{1}\left(\Gamma_{\omega}\right)$ with support in a fixed compact set. The constant $\gamma$ may depend on this compact set and the angle $\omega$, but not on $v$.

Proof. We proceed analogously to the proof of Gårding's inequality for $1+K$ in [11]. The operator $K$ maps even and odd functions on $\Gamma_{\omega}$ to even and odd functions, respectively. Therefore, it suffices to show

$$
\operatorname{Re}\left\langle D v, D\left(1 \pm K_{\omega}\right) v\right\rangle_{L^{2}\left(\mathbf{R}_{+}\right)} \geqslant \gamma\|v\|_{H^{1}\left(\mathbf{R}_{+}\right)}^{2}
$$

for all $v \in C_{0}^{\infty}[0, \infty)$ with

$$
K_{\omega} \phi(x):=\frac{1}{\pi} \int_{0}^{\infty} \operatorname{Im}\left(\frac{1}{x e^{i \omega}-y}\right) \phi(y) d y .
$$

By the Parseval relation for the Mellin transform we obtain

$$
\begin{aligned}
\operatorname{Re}\left\langle D v, D\left(1 \pm K_{\omega}\right) v\right\rangle & \\
=\frac{1}{2 \pi} \operatorname{Re} & \int_{\operatorname{Im} \lambda=-1 / 2}|\lambda+i|^{2} \\
& \cdot\left(1 \mp i \frac{\sinh (\pi-\omega)(\lambda+i)}{\sinh \pi(\lambda+i)}\right)|\hat{v}(\lambda+i)|^{2} d \lambda
\end{aligned}
$$

Here the Mellin transform is defined by

$$
\hat{v}(\lambda):=\int_{0}^{\infty} x^{i \lambda-1} v(x) d x
$$

and we use

$$
\begin{gathered}
\widehat{D v}(\lambda)=-i(\lambda+i) \hat{v}(\lambda+i) \\
\widehat{K_{\omega} v}(\lambda)=-i \frac{\sinh (\pi-\omega) \lambda}{\sinh \pi \lambda} \hat{v}(\lambda) \quad \text { for } \operatorname{Im} \lambda \in(-1,1)[11]
\end{gathered}
$$


Now we have

$$
\left|\frac{\sinh (\pi-\omega) \lambda}{\sinh \pi \lambda}\right| \leqslant\left|\sin \frac{\pi-\omega}{2}\right|=: 1-q<1 \quad \text { for all } \lambda \text { with } \operatorname{Im} \lambda=\frac{1}{2} .
$$

(In [11] we used this estimate for $\operatorname{Im} \lambda=-\frac{1}{2}$.) Therefore, we can estimate (2.8) from below by

$$
\frac{1-q}{2 \pi} \int_{\operatorname{Im} \lambda=-1 / 2}|\lambda+i|^{2}|\hat{v}(\lambda+i)|^{2} d \lambda=(1-q)\|D v\|_{L^{2}\left(\mathbf{R}_{+}\right)}^{2} \geqslant \gamma\|v\|_{H^{1}\left(\mathbf{R}_{+}\right)}^{2} .
$$

Proof of Lemma 2.1. Choose a partition of unity $\chi_{j} \in C_{0}^{\infty}\left(\mathbf{R}^{2}\right)$ with $\sum_{j=1}^{m} \chi_{j} \equiv 1$ on $\Gamma$ such that $\chi_{j} \geqslant 0$ and $\tilde{\chi}_{j}:=\sqrt{\chi_{j}} \in C_{0}^{\infty}$ and such that the support of $\chi_{j}$ contains exactly one corner $z_{j}$. (Thus $m=J$.) Then $a_{j}=b_{j}=b_{j}^{\prime}$ in Lemma 1.4 is the operator of multiplication by $\tilde{\chi}_{j}$. Then the commutator of $A$ with $a_{j}$ is compact on $H^{1}(\Gamma)$ (actually it maps $L^{2}(\Gamma)$ into $H^{1}(\Gamma)$ continuously), and the commutator of $Q$ with $a_{j}$ maps $H^{1}(\Gamma)$ into $L^{2}(\Gamma)$ and hence is compact from $H^{1}(\Gamma)$ to $H^{-1}(\Gamma)$. Together with Lemma 2.2, we see that all assumptions of Lemma 1.4 are satisfied, and its conclusion (1.10) gives (2.5).

We can now apply Lemma 1.1 and find immediately

THEOREM 2.3. There is an $N_{0} \in \mathbf{N}$ such that for all $N \geqslant N_{0}$ and all $f \in H^{1}(\Gamma)$ the system of collocation equations (0.5) has a unique solution $u_{N} \in S^{1}\left(\Delta_{N}\right)$. There is a constant $C$ independent of $N$ and $u$ such that

$$
\left\|u_{N}\right\|_{H^{1}(\Gamma)} \leqslant C\|u\|_{H^{1}(\Gamma)}
$$

and

$$
\left\|u-u_{N}\right\|_{H^{1}(\Gamma)} \leqslant C \inf \left\{\|u-\tilde{u}\|_{H^{1}(\Gamma)} \mid \tilde{u} \in S^{1}\left(\Delta_{N}\right)\right\}
$$

Remark 2.4. Note that the grids $\Delta_{N}$ need not be uniform. Nor do they have to include the corner points. One can conjecture that the estimates in Theorem 2.3 hold for arbitrary bounded Lipschitz domains, probably also in higher dimensions. Up to now, however, there exists no proof avoiding the use of Mellin transformation.

3. The First-Kind Integral Equation. We consider the collocation scheme (0.6) for the integral equation (0.2). The natural choice $Q=D^{2} ; X=H^{+1 / 2}(\Gamma), A=V$ is not useful, because in general then $\langle Q v, A v\rangle=\infty$, i.e., $Q$ does not map into $Y^{\prime}=(A X)^{\prime} \varsubsetneqq H^{-3 / 2}(\Gamma)$. We present two variations of this natural choice. Let us first define

$$
\stackrel{\circ}{H}^{1 / 2}(\Gamma):=\left\{u \in H^{1 / 2}(\Gamma) \mid \tilde{u}_{j} \in H^{1 / 2}(\Gamma)(j=1, \ldots, J),\right.
$$

$$
\text { where } \left.\tilde{u}_{j}=u \text { on } \Gamma^{j}, \tilde{u}_{j}=0 \text { on } \Gamma \backslash \Gamma^{j}\right\} .
$$

The norm in $\stackrel{\circ}{H}^{1 / 2}(\Gamma)$ is

$$
\|u\|_{H^{1 / 2}(\Gamma)}^{2}:=\sum_{j=1}^{J}\left\|u_{j}\right\|_{H^{1 / 2}(\Gamma)}^{2} .
$$

Then $\stackrel{\circ}{H}^{1 / 2}(\Gamma)$ is the completion of $C_{0}^{\infty}\left(\Gamma \backslash\left\{z_{1}, \ldots, z_{J}\right\}\right)$ in this norm. $\stackrel{\circ}{H}^{1 / 2}(\Gamma)$ is densely embedded in $H^{1 / 2}(\Gamma)$. The operator $V$ maps $\stackrel{H}{H}^{1 / 2}(\Gamma)$ continuously into $H^{3 / 2}(\Gamma)$ but it is not surjective, as the $H^{-1 / 2}(\Gamma)$-solution $u$ of Eq. (0.2) with smooth 
$f$ in general behaves like $O\left(\left|z-z_{j}\right|^{\alpha_{j}-1}\right)$ near $z_{j}$, where $\alpha_{j}$ was defined in the introduction [11]. Thus, in general, $u \notin H^{s}(\Gamma)$ for $s \geqslant \alpha_{0}-\frac{1}{2} \in\left(0, \frac{1}{2}\right)$. We define

$$
\stackrel{\circ}{S}\left(\Delta_{N}\right):=\left\{v \in S^{1}\left(\Delta_{N}\right) \mid v\left(z_{j}\right)=0, j=1, \ldots, J\right\},
$$

and we assume that

$$
\left\{z_{1}, \ldots, z_{J}\right\} \subset \Delta_{N}
$$

Thus, $\operatorname{dim} \stackrel{\circ}{S}\left(\Delta_{N}\right)=N-J$. Now choose $J$ functions $\eta_{1}, \ldots, \eta_{J} \in H^{3 / 2}(\Gamma)$ such that $\eta_{j}\left(z_{k}\right)=\delta_{j k}(j, k=1, \ldots, J)$. Define the projection operator $R: H^{3 / 2}(\Gamma) \rightarrow$ $H^{3 / 2}(\Gamma) \cap \stackrel{\circ}{H}^{1 / 2}(\Gamma)$ by

$$
R g(z):=g(z)-\sum_{j=1}^{J} g\left(z_{j}\right) \eta_{j}(z)
$$

Then the adjoint operator $R^{\prime}$ acts in $S^{-1}\left(\Delta_{N}\right)$ as follows:

$$
R^{\prime} \delta\left(z-x_{k}\right)=\delta\left(z-x_{k}\right)-\sum_{j=1}^{J} \eta_{j}\left(x_{k}\right) \delta\left(z-z_{j}\right) \quad\left(x_{k} \in \Delta_{N}\right)
$$

If we take the $(N-J)$-dimensional range space of $R^{\prime}$ as space $T_{N}$ of test functions, we arrive at the modified collocation equations

$$
\begin{aligned}
V u_{N}\left(x_{n}\right)-\sum_{j=1}^{J} V u_{N}\left(z_{j}\right) \eta_{j}\left(x_{n}\right)=f\left(x_{n}\right)- & \sum_{j=1}^{J} f\left(z_{j}\right) \eta_{j}\left(x_{n}\right) \\
& (n=1, \ldots, N), u_{N} \in \stackrel{\circ}{S}\left(\Delta_{N}\right) .
\end{aligned}
$$

These are satisfied by solutions in $\stackrel{\circ}{S}\left(\Delta_{N}\right)$ of $(0.6)$ but not conversely. Now we have the following situation:

$$
\begin{gathered}
X_{0}=X=\stackrel{\circ}{H}^{1 / 2}(\Gamma) ; \quad A=V ; \quad Y=A X ; \\
V_{N}=\stackrel{\circ}{S}\left(\Delta_{N}\right) ; \quad T_{N}=R^{\prime} S^{-1}\left(\Delta_{N}\right) ; \quad Q=R^{\prime} D^{2} .
\end{gathered}
$$

Furthermore, we can assume that $\eta_{j} \notin Y$ for $j=1, \ldots, J$. This implies (1.8). Then it is easily seen that the assumptions (i)-(iv) of Lemma 1.1 are satisfied. It remains to show the inequality (1.4).

LEMMA 3.1. There is a compact operator $C: \stackrel{\circ}{H}^{1 / 2}(\Gamma) \rightarrow \stackrel{\circ}{H}^{1 / 2}(\Gamma)^{\prime}$ and a constant $\gamma>0$ such that

$$
\operatorname{Re}(\langle D v, D R V v\rangle+\langle C v, v\rangle) \geqslant \gamma\|v\|_{\dot{H}^{1 / 2}(\Gamma)}^{2} \quad \text { for all } v \in \stackrel{H}{H}^{1 / 2}(\Gamma) .
$$

Proof. By localization to the reference angle $\Gamma_{\omega}$, decomposition into even and odd parts, and density arguments, we see that we have to show

$$
\operatorname{Re}\left(\left\langle D v, D\left(V_{0} \pm V_{\omega}\right) v\right\rangle+\langle C v, v\rangle\right) \geqslant \gamma\|v\|_{\tilde{H}^{1 / 2}\left(\mathbf{R}_{+}\right)}^{2}
$$

for all $v \in C_{0}^{\infty}\left(\mathbf{R}_{+}\right)$with support in a fixed compact set. Here,

$$
V_{\omega} \phi(x):=-\frac{1}{\pi} \int_{0}^{\infty} \log \left|1-\frac{x}{y} e^{-i \omega}\right| \phi(y) d y .
$$


Mellin transformation gives [11]

$$
\widehat{V_{\omega} \phi}(\lambda)=\frac{\cosh (\pi-\omega) \lambda}{\lambda \sinh \pi \lambda} \hat{\phi}(\lambda-i) \quad(\operatorname{Im} \lambda \in(0,1)) .
$$

By the Parseval relation we obtain

$$
\begin{aligned}
\left\langle D v, D\left(V_{0} \pm V_{\omega}\right) v\right\rangle & \\
=\frac{1}{2 \pi} \int_{\operatorname{Im} \lambda}=-1 / 2 & |\lambda+i|^{2} \overline{\hat{v}(\lambda+i)} \\
& \cdot\left(\frac{\cosh \pi(\lambda+i) \pm \cosh (\pi-\omega)(\lambda+i)}{(\lambda+i) \sinh \pi(\lambda+i)}\right) \hat{v}(\lambda) d \lambda
\end{aligned}
$$

For $\operatorname{Im} \lambda=-\frac{1}{2}$ we have $\overline{\hat{v}(\lambda+i)}=\overline{\hat{v}(\bar{\lambda})}$ and $\overline{\lambda+i}=\lambda$. Shifting the path of integration to $\operatorname{Im} \lambda=0$, we thus obtain

$$
\begin{aligned}
\langle D v, D( & \left.\left.V_{0} \pm V_{\omega}\right) v\right\rangle \\
& =\frac{1}{2 \pi} \int_{\operatorname{Im} \lambda=0} \lambda \frac{\cosh \pi(\lambda+i) \pm \cosh (\pi-\omega)(\lambda+i)}{\sinh \pi(\lambda+i)}|\hat{v}(\lambda)|^{2} d \lambda \\
& =\frac{1}{2 \pi} \int_{-\infty}^{\infty} m_{ \pm}(\lambda)|\hat{v}(\lambda)|^{2} d \lambda,
\end{aligned}
$$

with

$$
m_{ \pm}(\lambda)=\frac{\lambda}{\sinh \pi \lambda}(\cosh \pi \lambda \mp \cos \omega \cosh (\pi-\omega) \lambda \pm i \sin \omega \sinh (\pi-\omega) \lambda) .
$$

Hence,

$$
\operatorname{Re} m_{ \pm}(\lambda) \geqslant \gamma\left(1+\lambda^{2}\right) \quad(\lambda \in \mathbf{R})
$$

and thus

$$
\begin{aligned}
\operatorname{Re}\left\langle D v, D\left(V_{0} \pm V_{\omega}\right) v\right\rangle & \geqslant \gamma \int_{-\infty}^{\infty}\left(1+\lambda^{2}\right)|\hat{v}(\lambda)|^{2} d \lambda \\
& \geqslant \gamma\|v\|_{\tilde{H}^{1 / 2}\left(\mathbf{R}_{+}\right)}^{2}
\end{aligned}
$$

This gives (3.4), and the lemma is thus proved if we note that $1-R$ is finite-dimensional and hence compact.

Lemma 1.1 is now applicable to (3.3) and gives

THEOREM 3.2. There are $N_{0} \in \mathbf{N}$ and $C \geqslant 0$ such that for all $f \in H^{3 / 2}(\Gamma)$ with $u:=V^{-1} f \in \stackrel{\circ}{H}^{1 / 2}(\Gamma)$ and for all $N \geqslant N_{0}$ there exists a unique solution $u_{N} \in \stackrel{\circ}{S}^{1}\left(\Delta_{N}\right)$ of (3.3) satisfying

$$
\left\|u_{N}\right\|_{\dot{H}^{1 / 2}(\Gamma)} \leqslant C\|u\|_{\dot{H}^{1 / 2}(\Gamma)}
$$

and

$$
\left\|u-u_{N}\right\|_{\dot{H}^{1 / 2}(\Gamma)} \leqslant C \inf \left\{\|u-\tilde{u}\|_{\dot{H}^{1 / 2}(\Gamma)} \mid \tilde{u} \in \stackrel{\circ}{S}^{1}\left(\Delta_{N}\right)\right\} .
$$

As the hypothesis $u \in \stackrel{\circ}{H}^{1 / 2}(\Gamma)$ excludes the interesting case of $u$ having corner singularities, the theorem is of limited applicability. We shall construct now a second modification of the original collocation scheme that will work for all weak solutions if the right-hand side is smooth enough. 
We choose a weight function $\rho \in C^{\infty}\left(\mathbf{R}^{2} \backslash\left\{z_{1}, \ldots, z_{J}\right\}\right)$ with

$$
\rho(z)=\left|z-z_{j}\right| \text { in a neighborhood of } z_{j}, \quad j=1, \ldots, J .
$$

We assume again that $\left\{z_{1}, \ldots, z_{J}\right\} \subset \Delta_{N}$ and define

$$
S_{\rho}^{1}\left(\Delta_{N}\right):=\frac{1}{\rho} \dot{S}^{1}\left(\Delta_{N}\right)=\left\{u \mid \rho u \in \dot{S}^{1}(\Delta)\right\} .
$$

If we define

$$
\stackrel{\circ}{S}^{-1}\left(\Delta_{N}\right):=\left\{\phi \in S^{-1}\left(\Delta_{N}\right) \mid \operatorname{supp} \phi \cap\left\{z_{1}, \ldots, z_{J}\right\}=\varnothing\right\},
$$

then

$$
\operatorname{dim} S_{\rho}^{1}\left(\Delta_{N}\right)=\operatorname{dim} \dot{\circ}^{-1}\left(\Delta_{N}\right)=N-J
$$

and $u \in S_{\rho}^{1}\left(\Delta_{N}\right)$ implies $\rho D^{2} \rho u \in \dot{S}^{-1}\left(\Delta_{N}\right)$. Thus we set $V_{N}:=S_{\rho}^{1}\left(\Delta_{N}\right)$; $T_{N}:=\dot{S}^{-1}\left(\Delta_{N}\right)$; and $Q:=\rho D^{2} \rho$. Then the collocation equations (1.2) are: Find $u_{N} \in S_{\rho}^{1}\left(\Delta_{N}\right)$ with

$$
V u_{N}\left(x_{n}\right)=f\left(x_{n}\right) \text { for all } x_{n} \in \Delta_{N} \backslash\left\{z_{1}, \ldots, z_{J}\right\} \text {. }
$$

We need the weighted Sobolev space

$$
H_{\rho}^{1 / 2}(\Gamma):=\frac{1}{\rho} H^{1 / 2}(\Gamma)=\left\{u \mid \rho u \in H^{1 / 2}(\Gamma)\right\}
$$

with the norm

$$
\|u\|_{H_{\rho}^{1 / 2}}:=\|\rho u\|_{H^{1 / 2}}
$$

Then we set

$$
X:=H_{\rho}^{1 / 2}(\Gamma) \quad \text { and } \quad X_{0}:=H_{\rho}^{1 / 2}(\Gamma) \cap H^{-1 / 2}(\Gamma)
$$

with the norm

$$
\|u\|_{X_{0}}^{2}:=\|u\|_{H_{\rho}^{1 / 2}}^{2}+\|u\|_{H^{1 / 2}(\Gamma)}^{2}
$$

With $A:=V$ and $Y:=A X$ the hypotheses of Lemma 1.1 are satisfied: (1.8) holds because $Y \subset H_{\text {loc }}^{3 / 2}\left(\Gamma \backslash\left\{z_{1}, \ldots, z_{J}\right\}\right)$ is a Hilbert space of functions continuous on $\Gamma \backslash\left\{z_{1}, \ldots, z_{J}\right\}$. Thus assumption (i) holds. Assumption (ii) is trivial. Concerning assumption (iii), we note that every $v \in S_{\rho}^{1}\left(\Delta_{N}\right)$ is continuous on each segment $\Gamma^{j}$ and is constant on one-sided neighborhoods of the corners. Thus $V_{N} \subset X_{0}$ holds. It remains to show the estimates (1.3) and (1.4). In order to use the local principle, Lemma 1.4 , one has to consider commutators of multiplication operators by $\chi \in C_{0}^{\infty}\left(\mathbf{R}^{2}\right)$ with $A$ and with $Q$ :

$$
\begin{gathered}
B^{1}:=Q \chi-\chi Q=\rho\left(\left(D^{2} \chi\right)+2(D \chi) D\right) \rho, \\
B^{0}:=A \chi-\chi A .
\end{gathered}
$$

Here one can assume that $\chi$ equals one or zero on a neighborhood of the corner points. But outside such neighborhoods, the spaces $X$ and $X_{0}$ coincide with $H^{1 / 2}(\tilde{\Gamma})$, where $\tilde{\Gamma}$ is a smooth curve, and for the latter case the corresponding compactness results are well known from the calculus of pseudodifferential operators. 
Therefore, we only consider the reference angle $\Gamma_{\omega}$ and the bilinear form

$$
b(v, w):=\langle D \rho v, D \rho V w\rangle_{L^{2}\left(\Gamma_{\omega}\right)},
$$

where $v, w \in C_{0}^{\infty}\left(\Gamma_{\omega}\right)$ with support in a fixed compact set, and $\rho(z)=|z|$ on the support of $v$ and $w$.

Lemma 3.3. For all $v, w \in C_{0}^{\infty}\left(\Gamma_{\omega}\right)$,

$$
\begin{gathered}
b(v, w)=b_{1}(v, w)+b_{2}(v, w) \text { with } \\
b_{1}(v, w)=\langle D \rho v, V D \rho w\rangle, \quad b_{2}(v, w)=\langle D \rho v, V w\rangle=-\overline{b_{2}(w, v)} .
\end{gathered}
$$

The following estimates hold with $\gamma>0$ and $M$ independent of $v, w$ :

$$
\begin{gathered}
\left|b_{1}(v, w)\right| \leqslant M\|v\|_{H_{\rho}^{1 / 2}\left(\Gamma_{\omega}\right)}\|w\|_{H_{\rho}^{1 / 2}\left(\Gamma_{\omega}\right)}, \\
\operatorname{Re}\left(b_{1}(v, v)+\langle C v, v\rangle\right) \geqslant \gamma\|v\|_{H_{\rho}^{1 / 2}\left(\Gamma_{\omega}\right)}^{2}
\end{gathered}
$$

with a one-dimensional operator $C$, and

$$
\left|b_{2}(v, w)\right| \leqslant M\|v\|_{H_{\rho}^{1 / 2}\left(\Gamma_{\omega}\right)}\left(\|w\|_{H_{\rho}^{1 / 2}\left(\Gamma_{\omega}\right)}+\|w\|_{H^{-1 / 2}\left(\Gamma_{\omega}\right)}\right) .
$$

Proof. By taking even and odd parts of $v$ and $w$, one reduces everything to the half axis $\mathbf{R}_{+}$(compare Lemma 3.1). One has

$$
\rho D V_{\omega}=V_{\omega} D \rho \quad \text { with }(\rho v)(x)=x v(x) \text { on } \mathbf{R}_{+} .
$$

From this, there follows (3.8). The estimates (3.9), (3.10) and (3.11) follow from known properties of the operator $V$ :

$$
\begin{gathered}
|\langle D \rho v, V D \rho w\rangle| \leqslant M\|D \rho v\|_{H^{-1 / 2}}\|D \rho w\|_{H^{-1 / 2}}, \\
\operatorname{Re}\langle D \rho v, V D \rho v\rangle \geqslant \gamma\|D \rho v\|_{H^{-1 / 2}}^{2}, \\
|\langle D \rho v, V w\rangle| \leqslant M\|D \rho v\|_{H^{-1 / 2}}\|w\|_{H^{-1 / 2}} .
\end{gathered}
$$

Remark 3.4. The estimates in the above lemma can also be proved by Mellin transformation. In this way one can see also that the anti-Hermitian part $b_{2}$ of the sesquilinear form (3.7) is not bounded with respect to the Hermitian part $b_{1}$. This fact forces us to introduce the different norms in $X$ and $X_{0}$. With $V_{0}, V_{\omega}$ as defined in (3.5), one finds on $\mathbf{R}_{+}$:

$$
\langle D \rho \phi, D \rho V \psi\rangle=\frac{1}{2 \pi} \int_{\operatorname{Im} \lambda=0} m_{ \pm}(\lambda) \overline{\hat{\phi}(\lambda-i)} \hat{\psi}(\lambda-i) d \lambda
$$

with

$$
m_{ \pm}(\lambda)=\left(\lambda^{2}+i \lambda\right) \frac{\cosh \pi \lambda \pm \cosh (\pi-\omega) \lambda}{\sinh \pi \lambda} .
$$

Here, $\operatorname{Re} m_{ \pm}(\lambda)$ corresponds to $b_{1}$, and $i \operatorname{Im} m_{ \pm}(\lambda)$ to $b_{2}$, and for small $\lambda$ the latter is not dominated by the first one, so that estimates (1.3) and (1.4) cannot hold simultaneously with only one norm (i.e., $X=X_{0}$ ), whatever this norm might be!

Now we can apply Lemma 1.1 and obtain the corresponding stability and error estimates. 
THEOREM 3.5. There exist $N_{0} \in \mathbf{N}$ and $C \geqslant 0$ such that for all $f \in H^{1 / 2}(\Gamma)$ with $u=V^{-1} f \in H^{-1 / 2}(\Gamma)$ satisfying $\rho u \in H^{1 / 2}(\Gamma)$ there exists a unique solution $u_{N} \in$ $S_{\rho}^{1}\left(\Delta_{N}\right)$ of (3.6) satisfying

$$
\left\|u_{N}\right\|_{H_{\rho}^{1 / 2}(\Gamma)} \leqslant C\left(\|u\|_{H^{1 / 2}(\Gamma)}+\|u\|_{H_{\rho}^{1 / 2}(\Gamma)}\right)
$$

and

$$
\left\|u-u_{N}\right\|_{H_{\rho}^{1 / 2}(\Gamma)} \leqslant C \inf \left\{\|u-\tilde{u}\|_{H^{-1 / 2}(\Gamma)}+\|u-\tilde{u}\|_{H_{\rho}^{1 / 2}(\Gamma)} \mid \tilde{u} \in S_{\rho}^{1}\left(\Delta_{N}\right)\right\} .
$$

Remark 3.6. The hypothesis is satisfied for all smooth $f ; f \in H^{s}(\Gamma)$ with $s>3 / 2$ is sufficient. The reason is that the singular parts of $u$ are of the form (near a corner $\left.z_{j}\right)$

$$
\left|z-z_{j}\right|^{\alpha} \log \left|z-z_{j}\right|^{k}
$$

with some $\alpha>-1$ (actually, $\alpha=m \alpha_{j}+n-1>-\frac{1}{2}$, where $m \in \mathbf{N}, n \in \mathbf{N}_{0}$, and $\alpha_{j}$ is defined in the introduction). Such functions are obviously contained in $H_{\rho}^{1 / 2}(\Gamma)$.

4. Orders of Convergence. From the error estimates in Theorems 2.3, 3.2, and 3.5, one can derive convergence orders simply by utilizing results on the orders of best spline approximations in the respective norms.

In Theorem 2.3 there appears the standard $H^{1}$-norm, and one can therefore apply the standard approximation property of piecewise linear splines:

$$
\inf \left\{\|u-\tilde{u}\|_{H^{1}(\Gamma)} \mid \tilde{u} \in S^{1}\left(\Delta_{N}\right)\right\} \leqslant C h^{\sigma}\|u\|_{H^{1+\sigma}(\Gamma)} \quad(0 \leqslant \sigma \leqslant 1)
$$

where $C$ does not depend on $h$ and $u \in H^{1+\sigma}(\Gamma)$. Here the grid $\Delta_{N}$ with maximal meshwidth $h$ need not satisfy any uniformity condition. In our case of the secondkind integral equation, we can apply (4.1) for

$$
\sigma<\alpha_{0}-\frac{1}{2} \in\left(0, \frac{1}{2}\right)
$$

if the right-hand side satisfies $f \in H^{1+\sigma}(\Gamma)$, because then also $u \in H^{1+\sigma}(\Gamma)$ holds.

It is well known that one can obtain also an order of convergence of $h^{1}$ as in the smooth case if one uses graded meshes ([9], [16], [26]). We shall study the effect of graded meshes only in the case of the first-kind integral equation where, due to the nonstandard norm, we have to prove the approximation result anyway.

For the integral equation of the second kind, we can use Aubin-Nitsche type duality estimates to derive error estimates in lower-order Sobolev norms and obtain higher orders of convergence in this way. For this purpose we need an approximation property of the test functions in the dual space.

Lemma 4.1. There exists $C \geqslant 0$ such that

$$
\inf \left\{\|t-\tau\|_{H^{-1}(\Gamma)} \mid \tau \in S^{-1}\left(\Delta_{N}\right)\right\} \leqslant C h\|t\|_{L^{2}(\Gamma)}
$$

for all $t \in L^{2}(\Gamma), h>0$.

Proof. Given $t \in L^{2}$, we have to construct $\tau \in S^{-1}\left(\Delta_{N}\right)$ such that

$$
|\langle t-\tau, \phi\rangle| \leqslant C h\|t\|_{L^{2}(\Gamma)}\|\phi\|_{H^{1}(\Gamma)}
$$

for all $\phi \in H^{1}(\Gamma)$. Clearly, we can assume that $\Gamma$ is the unit interval and $t$ and $\phi$ are smooth, and

$$
\tau(x)=\sum_{j=1}^{J} c_{j} \delta\left(x-x_{j}\right) ; \quad 0=x_{1} \leqslant \cdots \leqslant x_{J}=1 .
$$


Now choose $c_{1}=0$, and

$$
c_{j}=\int_{x_{j-1}}^{x_{j}} t(\xi) d \xi \quad(j=2, \ldots, J) .
$$

Then by partial integration

$$
\langle t-\tau, \phi\rangle=-\sum_{j=2}^{J} \int_{x_{j-1}}^{x_{j}} \phi^{\prime}(x) \int_{x_{j-1}}^{x} t(\xi) d \xi d x,
$$

hence

$$
\begin{aligned}
|\langle t-\tau, \phi\rangle|^{2} & \leqslant\left(\sum_{j=2}^{J}\left\{\int_{x_{j-1}}^{x_{j}}\left|\phi^{\prime}(x)\right|^{2} d x \int_{x_{j-1}}^{x_{j}}\left|\int_{x_{j-1}}^{x} t(\xi) d \xi\right|^{2} d x\right\}^{1 / 2}\right)^{2} \\
& \leqslant \sum_{j=2}^{J} \int_{x_{j-1}}^{x_{j}}\left|\phi^{\prime}(x)\right|^{2} d x \sum_{j=2}^{J}\left|x_{j}-x_{j-1}\right|^{2} \int_{x_{j-1}}^{x_{j}}|t(x)|^{2} d x \\
& \leqslant\|\phi\|_{H^{1}(\Gamma)}^{2} h^{2}\|t\|_{L^{2}(\Gamma)}^{2} .
\end{aligned}
$$

THEOREM 4.2. Let $0 \leqslant \sigma \leqslant \alpha_{0}-1 / 2$ and $s \in[0,1]$. Then there is $C \geqslant 0$ such that for all $f \in H^{1+\sigma}(\Gamma)$ and the solution $u_{N}$ of the collocation equations $(0.5)$ there holds

$$
\left\|u-u_{N}\right\|_{H^{s}(\Gamma)} \leqslant C h^{1+\sigma-s}\|f\|_{H^{1+\sigma}(\Gamma)} .
$$

Proof. For $s=1$ this follows from Theorem 2.3 and (4.1). If we show it for $s=0$, then the general case will follow by interpolation. Thus we have to estimate

$$
\left\|u-u_{N}\right\|_{L^{2}(\Gamma)}=\sup \left\{\left|\left\langle l, u-u_{N}\right\rangle\right| \mid l \in L^{2}(\Gamma),\|l\|_{L^{2}(\Gamma)}=1\right\} .
$$

Now $1+K: H^{s}(\Gamma) \rightarrow H^{s}(\Gamma)$ is bijective and continuous for all $s \in[-1,1]$. The same holds for the adjoint operator $1+K^{\prime}$. Thus $\|l\|_{L^{2}}=1$ implies $t:=\left(1+K^{\prime}\right)^{-1} l$ $\in L^{2}(\Gamma)$ and $\|t\|_{L^{2}(\Gamma)} \leqslant M:=\left\|\left(1+K^{\prime}\right)^{-1}\right\|$. Now we use the definition of $u_{N}$ :

$$
\left\langle\tau,(1+K)\left(u-u_{N}\right)\right\rangle=0 \text { for all } \tau \in S^{-1}\left(\Delta_{N}\right) \text {. }
$$

Thus, for all $\tau \in S^{-1}\left(\Delta_{N}\right)$,

$$
\begin{aligned}
\left|\left\langle l, u-u_{N}\right\rangle\right| & =\left|\left\langle t,(1+K)\left(u-u_{N}\right)\right\rangle\right|=\left|\left\langle t-\tau,(1+K)\left(u-u_{N}\right\rangle\right\rangle\right| \\
& \leqslant\|t-\tau\|_{H^{-1}(\Gamma)} C\left\|\left(u-u_{N}\right)\right\|_{H^{1}(\Gamma) .}
\end{aligned}
$$

By Lemma 4.1,

$$
\left|\left\langle l, u-u_{N}\right\rangle\right| \leqslant C h\|t\|_{L^{2}(\Gamma)}\left\|u-u_{N}\right\|_{H^{1}(\Gamma)} .
$$

Hence,

$$
\left\|u-u_{N}\right\|_{L^{2}(\Gamma)} \leqslant C h\left\|u-u_{N}\right\|_{H^{1}(\Gamma)}
$$

and the result follows.

Remark 4.3. For $s>1 / 2$ we have, by Sobolev's embedding, estimates in Hölder norms. In particular, we have the pointwise estimate

$$
\left\|u-u_{N}\right\|_{L^{\infty}(\Gamma)}=O\left(h^{\alpha_{0}-\varepsilon}\right) \text { for any } \varepsilon>0,
$$


which can be improved (without further work!) to a $O\left(h^{3 / 2-\varepsilon}\right)$-estimate for suitably graded meshes.

Now we consider the case of the first-kind integral equation, Theorem 3.5. Spline approximation in weighted Sobolev spaces has been studied by several authors [6], [9], [15], but the kind of result needed here is not available. We assume that $u \in H^{-1 / 2}(\Gamma)$ is the solution of the equation $V u=f$ with $f$ smooth enough. Then $u$ has the following properties:

(i) $u \in H^{\alpha_{0}-1 / 2-\varepsilon}(\Gamma) ; \rho u \in H^{\alpha_{0}+1 / 2-\varepsilon}($ any $\varepsilon>0)$;

(ii) $\rho u=O\left(\rho^{\alpha_{0}}\right) ; D \rho u=O\left(\rho^{\alpha_{0}-1}\right) ; D^{2} \rho u=O\left(\rho^{\alpha_{0}-2}\right)$ near the corner points.

For these estimates on $u$ and $D \rho u, f \in H^{5 / 2+\varepsilon}(\Gamma)$ is sufficient, and for the estimate on $D^{2} \rho u, f \in H^{7 / 2+\varepsilon}(\Gamma)$ suffices. We approximate $u$ by its interpolation $\tilde{u}$ in $S_{\rho}^{1}(\Delta)$. Thus, if we define

$$
w:=\rho u, \quad \tilde{w}:=\rho \tilde{u},
$$

then $\tilde{w}$ is the piecewise linear interpolant of $w$ with nodes in $\Delta_{N}$. Note that $w$ is continuous on $\Gamma$ and vanishes at the corners. Now for the error estimate it suffices to consider a neighborhood of the corner points, because outside such a neighborhood, $u$ is smooth and therefore one has an approximation of order $h^{3 / 2}$ in the $H^{1 / 2}$-norm.

We consider a one-sided neighborhood of one corner point and assume that it is parametrized by the unit interval. We further assume that

$$
\rho(x)=x,
$$

and $u$ is given on $[0,1]$ with the properties (i) and (ii) above. We define $\tilde{u}, w$ and $\tilde{w}$ as above and write $\alpha:=\alpha_{0} \in\left(\frac{1}{2}, 1\right)$. We assume that the grid $\Delta_{N}$ has the form

$$
x_{j}=(j h)^{\beta}, \quad j=0, \ldots, N=1 / h
$$

with some $\beta \geqslant 1$. We have to estimate the two norms on $[0,1]$,

$$
\|u-\tilde{u}\|_{H^{-1 / 2}} \text { and }\|w-\tilde{w}\|_{H^{1 / 2}} .
$$

LEMMA 4.4. There is a constant $C$ such that

$$
\|u-\tilde{u}\|_{H^{-1 / 2}}+\|w-\tilde{w}\|_{H^{1 / 2}} \leqslant \begin{cases}C h^{3 / 2} & \text { for } \beta>\frac{2}{2 \alpha-1} . \\ C h^{\alpha \beta-\varepsilon} & \text { for } 1 \leqslant \beta<\frac{4}{2 \alpha+1}(\varepsilon>0) .\end{cases}
$$

Proof. From the estimates (ii) above we find for $x \in\left[x_{j}, x_{j+1}\right]$ :

$$
|(w-\tilde{w})(x)| \leqslant\left|x_{j+1}-x_{j}\right|^{2} \sup _{\xi \in\left[x_{j}, x_{j+1}\right]}\left|w^{\prime \prime}(\xi)\right| \leqslant C h^{\alpha \beta}(j+1)^{\alpha \beta-2}
$$

and

$$
\begin{aligned}
\left|(w-\tilde{w})^{\prime}(x)\right| & \leqslant\left|x_{j+1}-x_{j}\right| \sup _{\xi \in\left[x_{j}, x_{j+1}\right]}\left|w^{\prime \prime}(\xi)\right| \\
& \leqslant C h^{(\alpha-1) \beta}(j+1)^{(\alpha-1) \beta-1} .
\end{aligned}
$$

Furthermore, for $j=0$, i.e., $x \in\left[0, x_{1}\right]$ we have

$$
\left|\frac{1}{x}(w-\tilde{w})(x)\right| \leqslant\left|\frac{w(x)}{x}\right|+\left|\frac{w\left(x_{1}\right)}{x_{1}}\right| \leqslant C\left(x^{\alpha-1}+h^{(\alpha-1) \beta}\right) .
$$


We then estimate the following four integrals:

$$
\begin{aligned}
& J_{j}^{1}:=\int_{x_{j}}^{x_{j+1}}|(w-\tilde{w})(x)|^{2} d x \leqslant C h^{(2 \alpha+1) \beta}(j+1)^{(2 \alpha+1) \beta-5}, \\
& J_{j}^{2}:=\int_{x_{j}}^{x_{j+1}}\left|(w-\tilde{w})^{\prime}(x)\right|^{2} d x \leqslant C h^{(2 \alpha-1) \beta}(j+1)^{(2 \alpha-1) \beta-3}, \\
& J_{j}^{3}:=\int_{x_{j}}^{x_{j+1}}\left|\frac{1}{x}(w-\tilde{w})(x)\right|^{2} d x \leqslant C h^{(2 \alpha-1) \beta}(j+1)^{(2 \alpha-1) \beta-5}, \\
& J_{j}^{4}:=\int_{x_{j}}^{x_{j+1}}\left|\frac{1}{x}(w-\tilde{w})(x)\right| d x \leqslant C h^{\alpha \beta}(j+1)^{\alpha \beta-3} .
\end{aligned}
$$

This gives

$$
\|w-\tilde{w}\|_{L^{2}}^{2}=\sum_{j=0}^{N-1} J_{j}^{1} \leqslant C h^{(2 \alpha+1) \beta} \sum_{j=1}^{N} j^{(2 \alpha+1) \beta-5} .
$$

Now the sum on the right-hand side is bounded for $(2 \alpha+1) \beta-5<-1$ and bounded by $C N^{(2 \alpha+1) \beta-4}=C h^{-(2 \alpha+1) \beta+4}$ for $(2 \alpha+1) \beta-5>-1$. Thus we get

$$
\|w-\tilde{w}\|_{L^{2}} \leqslant\left\{\begin{array}{c}
C h^{2} \\
C h^{(\alpha+1 / 2) \beta}
\end{array}\right\} \text { for } \beta\left\{\begin{array}{l}
> \\
<
\end{array}\right\} \frac{4}{2 \alpha+1} .
$$

Analogously, we find, using $J_{j}^{2}$,

$$
\left\|(w-w)^{\prime}\right\|_{L^{2}}^{2} \leqslant C\left\{\begin{array}{c}
h \\
h^{(\alpha-1 / 2) \beta}
\end{array}\right\} \quad \text { for } \beta\left\{\begin{array}{l}
> \\
<
\end{array}\right\} \frac{2}{2 \alpha-1} .
$$

With $J_{j}^{3}$, we get

$$
\left\|\frac{1}{x}(w-\tilde{w})\right\|_{L^{2}}=\|u-\tilde{u}\|_{L^{2}} \leqslant C\left\{\begin{array}{c}
h^{2} \\
h^{(\alpha-1 / 2) \beta}
\end{array}\right\} \text { for } \beta\left\{\begin{array}{l}
> \\
<
\end{array}\right\} \frac{4}{2 \alpha-1} .
$$

With $J_{j}^{4}$, we get

$$
\left\|\frac{1}{x}(w-\tilde{w})\right\|_{L^{1}}=\|u-\tilde{u}\|_{L^{1}} \leqslant C\left\{\begin{array}{c}
h^{2} \\
h^{\alpha \beta}
\end{array}\right\} \text { for } \beta\left\{\begin{array}{c}
> \\
<
\end{array}\right\} \frac{2}{\alpha} .
$$

Now we use interpolation to get the desired norms:

$$
\|w-\tilde{w}\|_{H^{1 / 2}} \leqslant C\|w-\tilde{w}\|_{L^{2}}^{1 / 2}\|w-\tilde{w}\|_{H^{1}}^{1 / 2} \leqslant C\left\{\begin{array}{c}
h^{3 / 2} \\
h^{\alpha \beta}
\end{array}\right\} \quad \text { for } \beta\left\{\begin{array}{l}
> \\
<
\end{array} \begin{array}{l}
2 /(2 \alpha-1) \\
4 /(2 \alpha+1)
\end{array} .\right.
$$

Note that for $\alpha \in\left(\frac{1}{2}, 1\right)$ :

$$
1<\frac{4}{2 \alpha+1}<\frac{2}{\alpha}<\frac{2}{2 \alpha-1}<\frac{4}{2 \alpha-1} .
$$

From the Sobolev embedding theorem $H^{1 / 2+\varepsilon} \subset L^{\infty}$ we obtain by duality $L^{1} \subset$ $H^{-1 / 2-\varepsilon}$, i.e.,

$$
\|u-\tilde{u}\|_{H^{-1 / 2-,} \leqslant C\|u-\tilde{u}\|_{L^{1}} \text { for any } \varepsilon>0 .}
$$

Hence we find for any $\varepsilon>0$

$$
\|u-\tilde{u}\|_{H^{-1 / 2}} \leqslant C_{\varepsilon}\|u-\tilde{u}\|_{L^{1}}^{1-\varepsilon}\|u-\tilde{u}\|_{L^{2}}^{\varepsilon} .
$$


This gives

$$
\|u-\tilde{u}\|_{H^{-1 / 2}} \leqslant C\left\{\begin{array}{l}
h^{2} \\
h^{2-\varepsilon} \\
h^{\alpha \beta-\varepsilon}
\end{array}\right\} \text { for }\left\{\begin{array}{l}
\beta>4 /(2 \alpha-1), \\
2 / \alpha<\beta<4 /(2 \alpha-1), \\
1 \leqslant \beta<2 / \alpha
\end{array}\right.
$$

The rate $3 / 2$ is obtained here for $\beta>3 \alpha / 2$ which is less than $2 /(2 \alpha-1)$. Of course, one could also write down from this the rates for $4 /(2 \alpha+1)<\beta<$ $2 /(2 \alpha-1)$.

Note finally that for the slightly larger norms $\|\cdot\|_{\tilde{H}^{-1 / 2}}$ and $\|\cdot\|_{\tilde{H}^{1 / 2}}$ the same estimates hold, because they have the same interpolation properties [20].

Combining Lemma 4.4 and Theorem 3.5, we obtain our final result, Theorem 4.5 below. We assume that the grid $\Delta_{N}$ locally in a fixed one-sided neighborhood of each corner point has the form (4.2) in a suitable coordinate representation.

THEOREM 4.5. There exists $N_{0} \in \mathbf{N}$ and $C \geqslant 0$ such that for all $f$ smooth enough and $N \geqslant N_{0}$, the solutions $u \in H^{-1 / 2}(\Gamma)$ of Eq. (0.2) and $u_{N} \in S_{\rho}^{1}\left(\Delta_{N}\right)$ of (3.6) satisfy

$$
\left\|u-u_{N}\right\|_{H_{\rho}^{1 / 2}(\Gamma)} \leqslant C \begin{cases}h^{3 / 2} & \text { if } \beta>2 /\left(2 \alpha_{0}-1\right), \\ h^{\alpha_{0} \beta-\varepsilon} & \text { if } 1 \leqslant \beta<4 /\left(2 \alpha_{0}+1\right) ;(\text { any } \varepsilon>0) .\end{cases}
$$

Thus, we find also here the same optimal order of $h^{3 / 2}$ that is valid for the $H^{1 / 2}$-norm in the case of a smooth curve, if only the mesh refinement is strong enough at the corners.

Fachbereich Mathematik

Technische Hochschule Darmstadt

Schlossgartenstr 7

D-6100 Darmstadt, Federal Republic of Germany

School of Mathematics

Georgia Institute of Technology

Atlanta, Georgia 30332

1. P. M. Anselone, Collectively Compact Operator Approximation Theory and Applications to Integral Equations, Prentice-Hall, Englewood Cliffs, N. J., 1971.

2. D. N. ARnOld \& W. L. Wendland, "On the asymptotic convergence of collocation methods," Math. Comp., v. 41, 1983, pp. 349-381.

3. D. N. ARNOLD \& W. L. Wendland, "The convergence of spline collocation for strongly elliptic equations on curves," Numer. Math., v. 47, 1985, pp. 317-341.

4. K. E. AtKinson, A Survey of Numerical Methods for the Solution of Fredholm Integral Equations of the Second Kind, SIAM, Philadelphia, Pa., 1976.

5. K. E. AtKinson \& F. R. DE Hoog, "Collocation methods for a boundary integral equation on a wedge," in Treatment of Integral Equations by Numerical Methods (C. T. H. Baker and B. F. Miller, eds.), Academic Press, New York, 1983.

6. I. BABUSKA \& M. B. Rosenzweig, "A finite element scheme for domains with corners," Numer. Math., v. 20, 1972, pp. 1-21.

7. C. T. H. BAKER, The Numerical Treatment of Integral Equations, Clarendon Press, Oxford, 1977.

8. G. BRUHN \& W. L. WENDlAND, "Über die näherungsweise Lösung von linearen Funktionalgleichungen," in Funktionalanalysis, Approximationstheorie, Numerische Mathematik (L. Collatz and H. Ehrmann, eds.), Birkhäuser, Basel, 1967.

9. G. A. Chandler \& I. G. Graham, "Product integration-collocation methods for non-compact integral operator equations." (To appear.)

10. M. Costabel, "Boundary integral operators on curved polygons," Ann. Mat. Pura Appl. (4), v. 133, 1983, pp. 305-326. 
11. M. Costabel \& E. P. StePhan, "Boundary integral equations for mixed boundary value problems in polygonal domains and Galerkin approximation," Mathematical Models and Methods in Mechanics, Banach Center Publications, vol. 15, PWN, Warsaw, 1985, pp. 175-251.

12. M. Costabel \& E. P. Stephan, "The method of Mellin transformation for boundary integral equations on curves with corners," Numerical Solution of Singular Integral Equations, IMACS, 1984, pp. 95-100.

13. M. Costabel \& E. P. Stephan, "A direct boundary integral equation method for transmission problems," J. Math. Anal. Appl., v. 106, 1985, pp. 367-413.

14. M. Costabel, E. P. Stephan \& W. L. Wendland, "On boundary integral equations of the first kind for the bi-Laplacian in a polygonal plane domain," Ann. Scuola Norm. Sup. Pisa (4), v. 10, 1983, pp. 197-241.

15. J. ElSCHNER, "Galerkin methods with splines for singular integral equations over $(0,1)$," Numer. . Math., v. 43, 1984, pp. 265-281.

16. I. C. Gohberg \& I. A. Feldman, Convolution Equations and Projection Methods for Their Solution, Transl. Math. Monographs, vol. 41, Amer. Math. Soc., Providence, R. I., 1974.

17. R. Hagen \& B. Silbermann, "A finite element collocation method for bisingular integral equations," Applicable Anal., v. 19, 1985, pp. 117-135.

18. S. Hildebrandt \& E. Wienholtz, "Constructive proofs of representation theorems in separable Hilbert space," Comm. Pure Appl. Math., v. 17, 1964, pp. 369-373.

19. M. A. KraSnOSElSKII, ET AL., Approximate Solution of Operator Equations, Noordhoff, Groningen, 1972.

20. J. L. Lions \& E. Magenes, Non-Homogeneous Boundary Value Problems and Applications, Vol. I, Springer-Verlag, Berlin and New York, 1972.

21. V. G. MAZ'ya \& T. O. Shaposhnikova, "Change of variables as an operator in a pair of Sobolev spaces," Vestnik Leningrad Univ. Math., v. 15, 1983, pp. 53-58.

22. S. Prössdorf, "Ein Lokalisierungsprinzip in der Theorie der Spline-Approximation und einige Anwendungen," Math. Nachr., v. 119, 1984, pp. 239-255.

23. S. Prössdorf \& B. Silbermann, Projektionsverfahren und die näherungsweise Lösung singulärer Gleichungen, Teubner-Verlag, Leipzig, 1977.

24. S. Prössdorf \& B. Silbermann, Gestörte Projektionsverfahren und einige ihrer Anwendungen, Abhandlungen der Akademie d. Wiss. der DDR, Abt. Mathematik, Nr. 6N, Akademie-Verlag, Berlin, 1978, pp. 229-237.

25. S. PrösSDORF \& J. ElSCHNER, "Finite element methods for singular integral equations on an interval," Engrg. Anal., v. 1, 1984, pp. 83-87.

26. S. PrösSDorf \& A. RathSFeld, “A spline collocation method for singular integral equations with piecewise continuous coefficients," Integral Equations Operator Theory, v. 7, 1984, pp. 536-560.

27. J. Saranen \& W. L. Wendland, "On the asymptotic convergence of collocation methods with spline functions of even degree," Math. Comp., v. 171, 1985, pp. 91-108.

28. G. Schmidt, “On spline collocation for singular integral equations," Math. Nachr., v. 111, 1983, pp. 177-196.

29. W. L. WENDLAND, "Boundary element methods and their asymptotic convergence," in Theoretical Acoustics and Numerical Techniques (P. Filippi, ed.), CISM Lectures 277, Springer, Wien-New York, 1983, pp. 135-216.

30. W. L. Wendland, “On the spline approximation of singular integral equations and one-dimensional pseudodifferential equations on closed curves," Numerical Solution of Singular Integral Equations, IMACS, 1984, pp. 113-119. 